\title{
Dictionary Based Renovation from Minuzie to Image
}

\author{
R. Preethika ${ }^{1}$, J. Naveen Kumar ${ }^{2}$ \\ ${ }^{1}$ ME-CSE, Kingston Engineering College, Vellore, India \\ ${ }^{2}$ Assistant Professor, Kingston Engineering College, Vellore, India
}

\begin{abstract}
The set of minuzie points is considered to be most unique features for fingerprint delineation and is largely applicator in fingerprint matching. It was believed that only minuzie points do not contain sufficient information to renovate the original fingerprint image from which minuzie was excerpt. However, recent studies have shown that it is indeed possible to renovate fingerprint images from their minuzie delineation. In this paper, the previous experience about fingerprint ridge structures is conceal in terms of orientation patch and continuous phase patch dictionaries to improve the fingerprint renovation. The orientation patch dictionary is used to renovate the orientation field from minuzie, while the continuous phase patch dictionary is used to renovate the ridge pattern.
\end{abstract}

Keywords: fingerprint renovation, orientation patch dictionary, continuous phase patch dictionary, minuzie set.

\section{Introduction}

Fingerprint witness plays an imperative role in criminal investigation. Since a person's fingerprints are unique and do not change during the course of their life, other visible human characteristics such as facial features tend to change with age, but fingerprints are relatively resolute. Barring injuries or surgery causing deep scarring, or diseases comparatively leprosy prejudicial the formative layers of erosion ridge skin, finger and palm print features have never been shown to change their unit relationship throughout the life of a person. So they can be used to quickly and efficiently confirm or contradict a person's identity. In addition, fingerprints can be collected at a crime scene or theft area and have the potential to link a series of crimes together, or to place a dubious at the scene. Fingerprint plays an equally important role in identifying evidence following harm such as a cyclone, earthquake, bombing or other attack.

The Unique Identification Authority of India provides the world's largest fingerprint database system, with over 820 million fingerprint, face and iris biometric database. India's Unique Identification project is also known as Aadhaar, semantics of the word "the foundation" in different Indian languages. Aadhaar is a voluntary program, with the ambitious goal of eventually providing constant national ID documents to most of India's 1.2 billion residents. Aadhaar database is larger than any other database in the world, Aadhaar's ability to drag automated fingerprint and iris modalities enables rapid and safe automated searching and identification impractical to accomplish with fingerprint technology alone, peculiarly when seeking children and elderly resident's fingerprints. Initially fingerprints are used only for salary details in company later we use fingerprints as evidence in crime and theft so renovation is needed because we can't get full fingerprint from the crime area so reconstruction is improved in forensics department .

\section{Objectives}

The main focus is to reconstruct the finger print for the theft purpose and to match the reconstructed finger print with original database (Aadhaar card). We also focus on reproducing the original fingerprint image from an input minutiae set.

\section{Existing System}

Existing reconstruction algorithm contains two main steps: 1) Orientation field reconstruction 2) Ridge pattern reconstruction. In Hill algorithm, the orientation field was renovation from the singular points (core and delta) using Zero pole model. The other step is ridge pattern reconstruction based on the orientation field. It generates only a partial skeleton of the fingerprint. This method is further improved in Ross algorithm, a set of minuzie triplets was proposed to renovate orientation field in triangles without using singular points. Linear integral convolution algorithm to impact texture-like appearance and low pass filtering used to get wider ridges. However it can only generate a partial fingerprint. Feng and Jain used nearest minutiae in eight sector algorithm and AM-FM model it results spurious minuzie and blocking effect appear in the reconstructed fingerprint. The reconstructed fingerprint image is not same as the original fingerprint image that the minuzie were extracted from. An important reason for loss of matching performance is that no previous knowledge about fingerprint ridge structure was utilized in these renovation approaches to reproduce the fingerprint characteristics.

\section{Literature Survey}

1) An Improved Scheme for Full Fingerprint Reconstruction:

The minutiae template does not contain sufficient information for reconstructing the original fingerprint. This paper proposes a scheme to reconstruct a full fingerprint image from the minutiae points based on the 
amplitude and frequency modulated (AM-FM) fingerprint model.

2) Algorithm for Detection and Elimination of False Minutiae in Fingerprint Images:

There are many false minutiae among the extracted minutiae. False minutiae will decrease the performance of the fingerprint identification system by increasing both FRR and FAR. Minutiae post processing algorithm is used to eliminate false minutiae without eliminating true minutiae

3) Reconstructing Fingerprints from Minutiae Points: The Technique: orientations of fingerprint ridges can be electively predicted using minutiae points. This can be beneficial in applications like smart cards (where memory is critical) since the orientation map required for matching need not be stored explicitly but can be generated from the template.

4) Fingerprint Verification System using Minutiae Extraction Technique:

Improved thinning in the present work contributes to: The image becomes perfectly thinned to single pixel width. More number of bifurcations can be detected, which were missed earlier due to the presence of erroneous pixels in the thinned image. False Minutiae Removal techniques contribute to: Twelve different false minutiae are detected and are removed properly. Probability of getting refined image free of erroneous minutiae will be more.

5) Latent Fingerprint Matching:

Poor quality of ridge impressions, small finger area, and large nonlinear distortion are the main difficulties in latent fingerprint matching compared to plain or rolled fingerprint matching. We propose a system for matching latent fingerprints found at crime scenes to rolled fingerprints enrolled in law enforcement databases. In addition to minutiae, using extended features, including singularity, ridge quality map, ridge flow map, ridge wavelength map, and skeleton are the most effective features in improving the matching accuracy.

6) Crowd Powered Latent Fingerprint Identification: Fusing AFIS with Examiner Markups:

These papers propose a synergistic crowd powered latent identification framework where multiple latent examiners and the AFIS work in conjunction with each other to boost the identification accuracy of the AFIS.

7) Latent Fingerprint Matching: Performance Gain via Feedback from Exemplar Prints:

This paper proposes incorporating feedback from exemplar (rolled or plain fingerprint) to refine feature extraction in latent with the eventual goal of improving the latent matching accuracy.

8) Recognizing Infants and Toddlers Using Fingerprints:

Increasing the Vaccination Coverage; Vaccinepreventable diseases continue to take a heavy toll on children in geographical regions and countries without high immunization coverage. For improving the immunization coverage, an effective immunization program needs to keep track of the vaccination schedule of children.
9) Fingerprint Image Reconstruction from Standard Templates:

This paper shows reconstructed images are very realistic and that, although it is unlikely that they can fool a human expert, there is a high chance to deceive state-of-the-art commercial fingerprint recognition systems.

10) Latent Fingerprint Indexing: Fusion of Level 1 and Level 2 Features:

This paper propose an indexing technique, primarily for latent, that combines multiple level 1 and level 2 features to filter out a large portion of the background database while maintaining the latent matching accuracy. Our approach consists of combining minutiae, singular points, and orientation field and frequency information.

11) Learning Fingerprint Reconstruction: From Minutiae to Image:

The proposed method for orientation field reconstruction only considers the local orientation pattern. The use of global orientation prior knowledge as well as singular points may further improve the ridge orientation reconstruction. Future work will investigate frequency field reconstruction directly from the minutiae position and direction.

12) A minutia-based partial fingerprint recognition system:

Matching incomplete or partial fingerprints continues to be an important challenge today, despite the advances made in fingerprint identification techniques. While the introduction of compact silicon chip-based sensors that capture only part of the fingerprint has made this problem important from a commercial perspective, there is also considerable interest in processing partial and latent fingerprints obtained at crime scenes

13) From Template to Image: Reconstructing Fingerprints from Minutiae Points:

This paper demonstrates that three levels of information about the parent fingerprint can be elicited from a given minutiae template: the orientation field, the fingerprint class, and the friction ridge structure. The orientation field is estimated using minutia triplet information. The class of the parent fingerprint is inferred from specific attributes of the minutiae distribution in conjunction with the estimated orientation field. The ridge structure of the parent fingerprint is generated by applying streamlines and LIC on the estimated orientation field..

14) Spurious Minutia Removal Technique using Euclidean distance approach:

The fingerprint images get degraded and corrupted due to variations in skin and impression conditions. Thus, image enhancement techniques are employed prior to minutiae extraction. A critical step in automatic fingerprint matching is to reliably extract minutiae from the input fingerprint images. This paper proposes the classification of false minutiae for better matching results. The fake minutia is rejected.

15) Risk of Masquerade Arising from the Storage of Biometrics:

This paper proposes zero pole model and partial reconstruction algorithm for fingerprint reconstruction. It results only partial skeleton of a fingerprint is obtained. 


\section{International Journal of Science and Research (IJSR) \\ ISSN (Online): 2319-7064 \\ Index Copernicus Value (2013): 6.14 | Impact Factor (2014): 5.611}

16) Ridge Orientation Estimation and Verification Algorithm for Fingerprint Enhancement:

To enhance the images use filtering techniques that can be categorized into isotropic and anisotropic according to the filter kernel. Isotropic filtering can properly preserve features on the input images but can hardly improve the quality of the images. On the other hand, anisotropic filtering can effectively remove noise from the image but only when a reliable orientation is provided. In this paper, we propose a ridge orientation estimation and verification algorithm which can not only generate an orientation of ridge flows, but also verify its reliability.

\section{Proposed System}

Our goal is to utilize a dictionary based approach to improve the fingerprint renovation from a given minuzie set. Two dictionaries are constructed for fingerprint reconstruction:

1) Orientation patch dictionary

2) Continuous phase patch dictionary.

These orientation patch dictionary is used to renovate the orientation field from a minuzie set. Continuous phase patch dictionary is used to reconstruct the ridge pattern. Instead of reconstructing ridge pattern phase and spiral phase globally, I propose to reconstruct fingerprint patches using continuous phase patch dictionary and minuzie belonging to these patches; these patches are selected to form a fingerprint image. The spurious minuzie detected in the phase of the reconstructed fingerprint image but not included in the input minuzie template, are then removed using global AF-FM model.

\section{Proposed Reconstruction Algorithm}

\section{A.Minuzie extraction:}

Minuzie is the set of ridge ending points and ridge bifurcations. it is distinctive features so it is easy to reconstruct the fingerprint image, moreover minuzie has less memory to save in database than whole fingerprint.Converting grayscale image into black and white to avoid confusion between ridge and valley.Extracting minuzie points from contrasting image.

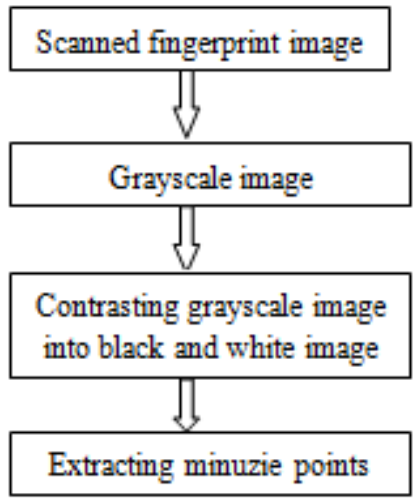

\section{B. Dictionary Construction}

Dictionary construction technique is proposed it makes easier to search the orientation field and ridge pattern .orientation field is used to reconstruct the fingerprint at system level, whereas ridge pattern is used to give the realistic image for fingerprint.

1) Orientation patch dictionary: The orientation patch dictionary proposed by feng et al. for latent enhancement is directly utilized as prior knowledge of ridge flow for orientation field reconstruction. The orientation patch dictionary contains number of orientation patches, is constructed from a set of high quality fingerprints.

2) Continuous phase patch dictionary: Continuous phase patch dictionary is used to reconstruct ridge pattern for giving the realistic fingerprint image. Auto correlation technique are used to constructing dictionaries each fingerprints are compared with remaining fingerprint grayscale image in database and calculate the probability of same pattern which is matches above $95 \%$ and constructing dictionary.
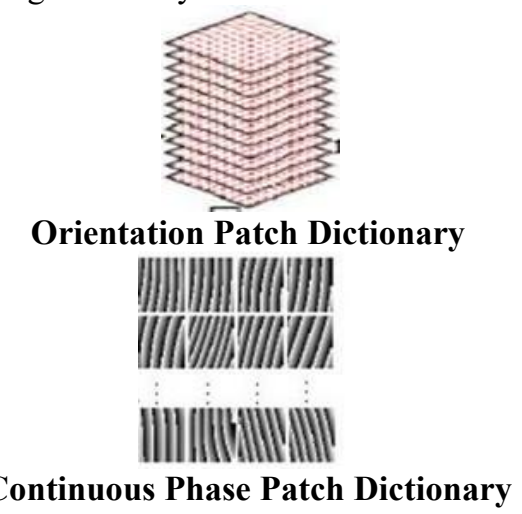

\section{Orientation field reconstruction}

The orientation field reconstructed by the orientation patch dictionary may also change the orientations in the blocks containing minuzie.

1) The orientations at the blocks with minuzie are replaced by the directions of minuzie.

2) The cosine and sine components of the double values of the orientations at the blocks without minuzie are smoothed using a Gaussian mask.

Compare to Feng and Jain algorithm the proposed algorithm is able to better orientation approximation, especially in the regions near singular points.

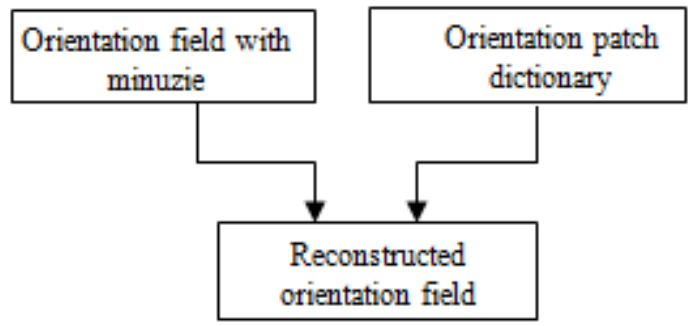

\section{Fingerprint Reconstruction}

The ridge patch dictionary is used to renovate fingerprint image patches based on the reconstructed orientation field and ridge frequency.

1) Fingerprint patch reconstruction: The continuous phase patch sub dictionary is selected based on the orientation similarity between orientation field and orientation patch centers. The minuzie can be added by combining the continuous phase patch and the spiral phase computed from 


\section{International Journal of Science and Research (IJSR) \\ ISSN (Online): 2319-7064 \\ Index Copernicus Value (2013): 6.14 | Impact Factor (2014): 5.611}

the minuzie in the patch. The set of minuzie points $m_{p}$ in the patch $p$ where $(\mathrm{x}, \mathrm{y})$ and $\mathrm{d}$ are the coordinates (location) and direction of the minuzie. There may be a few spurious minuzie in the reconstructed fingerprints that are introduced in the overlapping regions because suboptimal selection may be obtained in minimizing the energy.

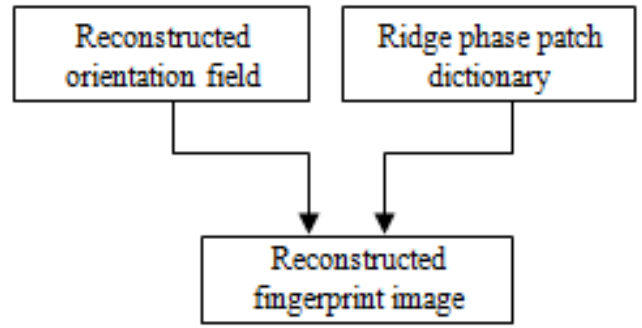

\section{E. Fingerprint Refinement}

It is the final stage of reconstructing fingerprint image, the spurious minuzie removed by using amplitude frequency model. After all discontinuity segments have been considered, Gabor filtering is used to smooth the fingerprint region around these discontinuity segments, the demodulation is used again to obtain the final phase image and then final reconstruction.

\begin{tabular}{|c|c|c|}
\hline $\begin{array}{c}\text { Reconstructed fingerprint } \\
\text { with false minuzie }\end{array}$ & $\begin{array}{c}\text { Reconstructed } \\
\text { fingerprint }\end{array}$ \\
\hline
\end{tabular}

\section{Conclusion}

Reconstruction is useful in where we can't get full fingerprint image, it is more useful in crime area and forensics department. Not only in crime area or theft it is also used in hospitals to find correct child of parent. In biometrics we have iris code, palm print, face recognition, fingerprint, bone, hair etc. But fingerprint is most distinctive features compared to others, even twins may be have identical DNA rarely but fingerprint doesn't have the same, around 64 billion people there is chance to have the same fingerprint but it's rare case. In this paper dictionary is used to reconstruct the fingerprint image to reduce the time for searching and save the memory by using minuzie points. At INTERPOL, we maintain databases of fingerprints and DNA profiles, allowing police across the world to make connectives between criminals and crime scenes. We also provide training to police in our member countries, to ensure that frontline officers have the knowledge and skills necessary to assess, preserve and share evidence in line with best practices. At INTERPOL, we manage a database of fingerprints, containing more than 189,000 fingerprint records. . India is collecting Unique Identification project is also known as Aadhaar.

\section{References}

[1] S. Liand A. C. Kot, “Attack using reconstructed fingerprint,"in Proc. IEEE Int. Workshop on Information Forensics and Security (WIFS), Foz do Iguacu, Brazil, Nov. 29-Dec. 2, 2011.

[2] A. M. Bazen, G. T. Verwaaijen, S. H. Gerez, L. P. Veelenturf, and B. J. Zwaag Vander, "A correlationbased fingerprint verification system,"'in Proc.Workshop on Circuits Systems and Signal Processing (ProRISC), 2000, pp. 205-213.

[3] J. Feng, Z. Ouyang, and A. Cai, "Fingerprint matching using ridges," Pattern Recognition., vol. 39, pp. 21312140, 2006.

[4] A. Jain, H. Lin, and R. Bolle, "On-line finger print verification, "IEEE Trans. Pattern Anal. Mach. Intell., vol. 19, no. 4, pp. 302-314, Apr. 1997.

[5] C.Hill, "Risk of Masquerade Arising From the Storage of Biometrics," Master's thesis, Australian National University, Australia,2001.

[6] A. Ross, J. Shah, and A. K. Jain, "From template to image: Reconstructing fingerprints from minutiae points,'IEEE Trans. Pattern Anal. Mach. Intell., vol. 29, no. 4, pp. 544-560, Apr. 2007.

[7] R. Cappelli, A. Lumini, D. Maio, and D. Maltoni, "Fingerprint image reconstruction from standard templates," IEEE Trans. Pattern Anal. Mach. Intell., vol. 29, no. 9, pp. 1489-1503, Sep. 2007.

\section{Author Profile}

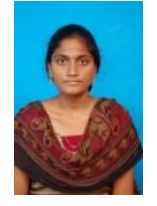

Preethika Rajan Has completed her graduation in B.E Computer Science and Engineering in the year of 2013 at Kingston engineering College. Affiliated by Anna University, Chennai, India. Presently doing her post graduation in M.E Computer Science and Engineering in the year 2014-16 at Kingston Engineering College.

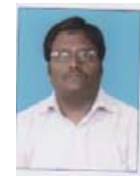

Naveen Kumar J, M.E (CSC), presently working as assistant professor in Kingston engineering college, Vellore. He is 8 years experienced professional in the field computer science and engineering. $\mathrm{He}$ has published a international journal "GROUP BASED POLICY IN SOCIAL NETWORKS" in the year of 